\title{
INTERSTELLAR SCATTERING AND THE \\ EINSTEIN RING PKS 1830-211
}

\author{
D.L. JONES, R.A. PRESTON, D.W. MURPHY AND D.L. MEIER \\ Jet Propulsion Laboratory, Mail Code 238-332, \\ 4800 Oak Grove Drive, Pasadena, CA. 91109, USA \\ AND \\ D.L. JAUNCEY, J.E. REYNOLDS AND A.K. TZIOUMIS \\ Australia Telescope National Facility, CSIRO, \\ PO Box 76, Epping 2121, NSW, Australia
}

\begin{abstract}
The remarkably strong radio gravitational lens PKS 1830211 consists of a one arcsecond diameter Einstein ring with two bright compact components located on opposite sides of the ring. We have obtained 22 $\mathrm{GHz}$ VLBA data on this source to determine the intrinsic angular sizes of the compact components. Previous VLBI observations at lower frequencies indicate that the brightness temperatures of these components are significantly lower than $10^{10} \mathrm{~K}$ (Jauncey et al. 1991), less than is typical for compact synchrotron radio sources and less than is implied by flux density variations. A possible explanation is that interstellar scattering is broadening the apparent angular size of the source and thereby reducing the observed brightness temperature. Our VLBA data support this hypothesis.
\end{abstract}

The position of PKS $1830-211$ is only a few degrees away from the galactic center $\left(b=-5.7^{\circ}, l=12.2^{\circ}\right)$. It is therefore plausible that significant interstellar scattering (ISS) within our galaxy may occur along this line of sight. The effects of ISS decrease rapidly with frequency, and should be small at $22 \mathrm{GHz}$.

We observed PKS 1830-221 with the full VLBA at $22 \mathrm{GHz}$ in May 1994. The data were correlated twice using phase centers corresponding to the locations of the two compact components, which we designate the northeast (NE) and southwest (SW) components. After calibration and fringe fitting in AIPS, we used the Caltech program Difmap for editing,

C. S. Kochanek and J. N. Hewitt (eds), Astrophysical Applications of Gravitational Lensing, 345-346. (c) 1996 IAU. Printed in the Netherlands. 

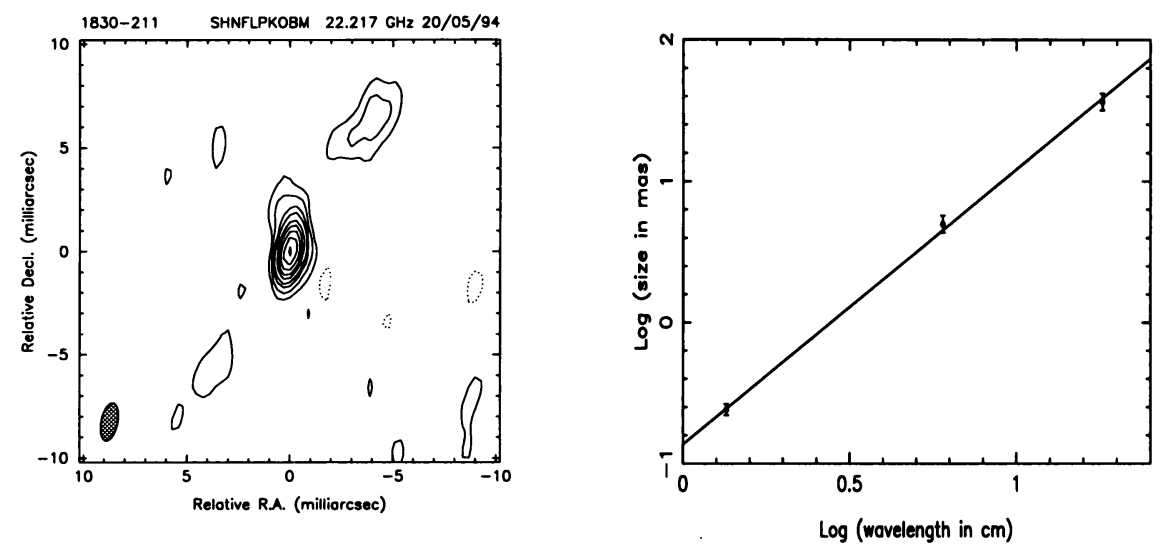

Figure 1. LEFT: The contours are $-2,2,5,10,15,25,50,70$, and $95 \%$, and the restoring beam FWHM is $1.9 \times 0.8$ mas with the major axis along position angle $-11.5^{\circ}$. The absolute flux density scale is not yet fully calibrated, but this does not affect the measured angular size of the core. RIGHT: The deconvolved minor axis angular sizes and their (formal) errors determined with the AIPS program IMFIT, using only the upper $50 \%$ or less of the brightness range to avoid bias by any extended low-level structure.

self-calibration, imaging, and deconvolution. This paper presents our image of the SW component; imaging of the NE component is in progress.

The two figures above show our VLBA image of the SW component of PKS 1830-211 and the deconvolved minor axis width of this component at three frequencies: $1.7 \mathrm{GHz}$ (unpublished data from an ad-hoc VLBA experiment in 1990), $4.9 \mathrm{GHz}$ (Jones 1994), and $22 \mathrm{GHz}$ (new data).

The slope of the line fit to the angular size measurements is $1.95 \pm 0.14$, consistent with the $\lambda^{2}$ dependence expected for scattering. This suggests that angular size measurements made by VLBI at frequencies $\leq 22 \mathrm{GHz}$ are indeed affected by angular broadening due to ISS. At $22 \mathrm{GHz}$ the size of the SW component core is $0.6 \times 0.2$ mas and $\mathrm{T}_{\mathrm{b}} \sim 10^{11} \mathrm{~K}$.

Acknowledgements: This research was carried out at the Jet Propulsion Laboratory, California Institute of Technology, under contract with NASA.

\section{References}

Jauncey, D.L., et al., 1991, Nature, 352, 132

Jones, D.L. 1994, in Compact Extragalactic Radio Sources, ed. J.A. Zensus \& K.I. Kellermann (Socorro: NRAO), 135 